\title{
An Atypical Localized Form of Hidradenitis Suppurativa of the Jawline and Neck Mimicking Severe Cystic Acne on Presentation
}

\author{
María Adriana Castrillón Velásquez ${ }^{\mathrm{a}, \mathrm{c}}$ Minhee Kim ${ }^{\mathrm{a}, \mathrm{b}}$ Mei-Heng Tan ${ }^{\mathrm{a}}$ \\ Kim Tran ${ }^{a, b}$ Dedee F. Murrella, \\ ${ }^{a}$ Department of Dermatology, St. George Hospital, and ${ }^{b}$ Faculty of Medicine, University of New South Wales, \\ Sydney, NSW, Australia; ' Clínica Alemana de Santiago, Facultad de Medicina Clínica Alemana-Universidad del \\ Desarrollo, Santiago, Chile
}

\section{Established Facts}

- Hidradenitis suppurativa (HS) can affect typical and atypical sites.

- When atypical sites are involved, there are usually typical areas compromised that facilitate the diagnosis.

\section{Novel Insights}

- Patients who present with nodules on the chin and neck which are resistant to acne therapies and develop sinuses may in fact have HS, and the chin and neck may be the only sites affected.

- Atypical HS may respond to TNF alpha inhibitors.

\section{Keywords}

Hidradenitis suppurativa - Atypical presentation - Severe cystic acne $\cdot$ Acne conglobata

\begin{abstract}
Hidradenitis suppurativa (HS) is a chronic and debilitating suppurative disease primarily affecting the axillae, perineum, and inframammary regions, where apocrine sweat glands are present. However, HS can occur in atypical locations. We present an interesting case of a 40-year-old man who developed chronic painful subcutaneous nodules, deep sinus tracts, and abscesses involving the jawline and the anterior aspect of the neck as the only parts of the body affected and who responded satisfactorily to adalimumab
\end{abstract}

\section{KARGER}

() 2017 S. Karger AG, Basel

E-Mail karger@karger.com

www.karger.com/sad and laser hair removal treatment. This case is relevant because it helps clinicians to remember that HS may be isolated to atypical locations, such as the anterior aspect of the neck and chin. It also supports another possible HS pathogenesis which consists of the occlusion of terminal hair follicles rather than being essentially a disorder of the apocrine glands.

(c) 2017 S. Karger AG, Basel

\section{Introduction}

Hidradenitis suppurativa (HS) is a chronic suppurative disease primarily affecting the axillae, perineum, and inframammary regions, where apocrine sweat glands are present. However, HS can occur in atypical locations $[1,2]$. 

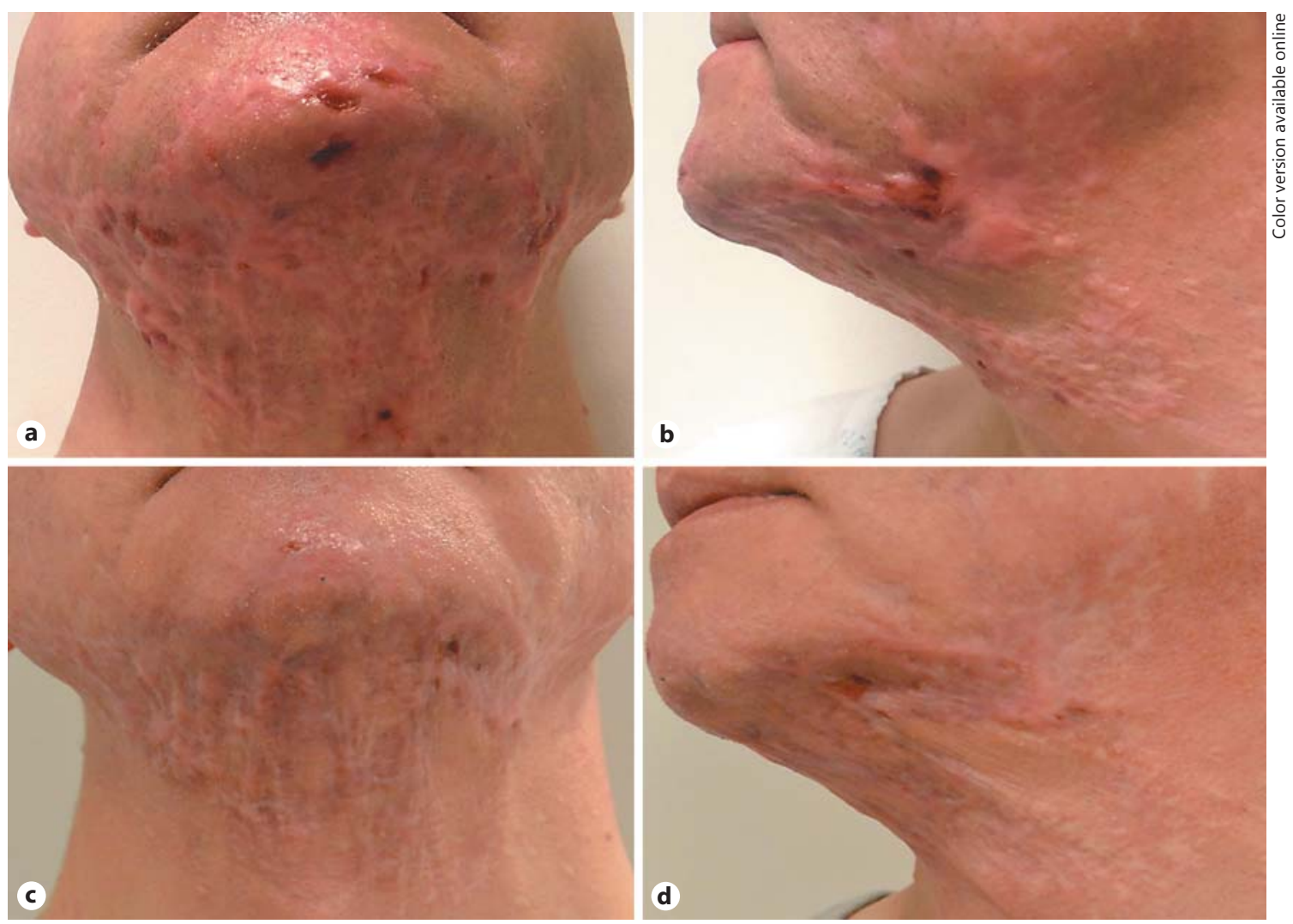

Fig. 1. Clinical features. a, b HS with rope-like hypertrophic scars within raised inflammatory plaques. Sartorius score: 70. c, d Less inflammation and fewer scars after 1 year of adalimumab and hair laser removal treatment. Sartorius score: 14 .

\section{Case Report}

A 40-year-old man presented for a second opinion with a 3 -year history of chronic painful subcutaneous nodules, deep sinus tracts, and abscesses involving the jawline and the anterior aspect of the neck. He had been previously managed as persistent adult acne. Initially, the lesions appeared to be folliculitis with trapped hairs, which later transformed into subcutaneous nodules that drained intermittently and healed with hypertrophic scars, simulating severe cystic acne. The patient had no personal or family history of acne vulgaris, HS, diabetes, or gastrointestinal illness. He had previously been treated with multiple courses of topical and oral antibiotics, which resulted in partial and temporary improvement.

On examination, there were numerous rope-like hypertrophic scars within raised inflammatory plaques, deep-seated nodules, sinuses, and fistulae with purulent and bloody discharge on the jawline and the anterior neck (Fig. 1a, b). There were no other skin lesions elsewhere. Punch biopsies for hematoxylin-eosin, fungal polymerase chain reaction, as well as fungal and bacterial cultures were performed. The hematoxylin-eosin biopsy showed an epidermis which was covered by a parakeratotic stratum corneum. There was extensive underlying fibrosis, and only isolated follicles remained. There were increased vessels present together with lym- phocytic inflammation and plasma cells as well as an area of ulceration covered by fibrin, locules of serum, and neutrophilic debris (Fig. 2a, b). All the tests were negative for any causative microorganisms. Isotretinoin $10 \mathrm{mg} /$ daily for 10 months was tried, with no improvement. Given the clinical features, the lack of a primary infectious disease, and the absent response to acne treatment, the diagnosis of HS in an atypical location was made. He was found to be insulin resistant as well. The insulin resistance was established by measuring serum insulin values before and after a $75 \mathrm{~g}$ oral glucose tolerance test. The insulin levels were $13 \mathrm{mU} / \mathrm{L}$ (normal $<10$ $\mathrm{mU} / \mathrm{L}$ ), $130 \mathrm{mU} / \mathrm{L}$ (normal $<60 \mathrm{mU} / \mathrm{L}$ ), and $44 \mathrm{mU} / \mathrm{L}$ (normal $<60$ $\mathrm{mU} / \mathrm{L}$ ) when fasting and 1 and $2 \mathrm{~h}$ after glucose challenge, respectively.

Considering the severity of the disease (Sartorius score 70 and Hurley stage 2) and the lack of response to other conventional treatments, biologic therapy with adalimumab $40 \mathrm{mg} /$ weekly was added initially to isotretinoin. Three months later, the patient improved significantly (Sartorius score 59) and isotretinoin was ceased. Subsequently, monthly laser treatment was added as an adjuvant therapy to avoid trapped hairs (long-pulsed Nd:YAG laser, 1,064 $\mathrm{nm}$ wavelength). After 1 year of ongoing treatment with adalimumab and laser treatment, the patient continued to improve, with a Sartorius score of 14 (Fig. 1c, d, 3). 

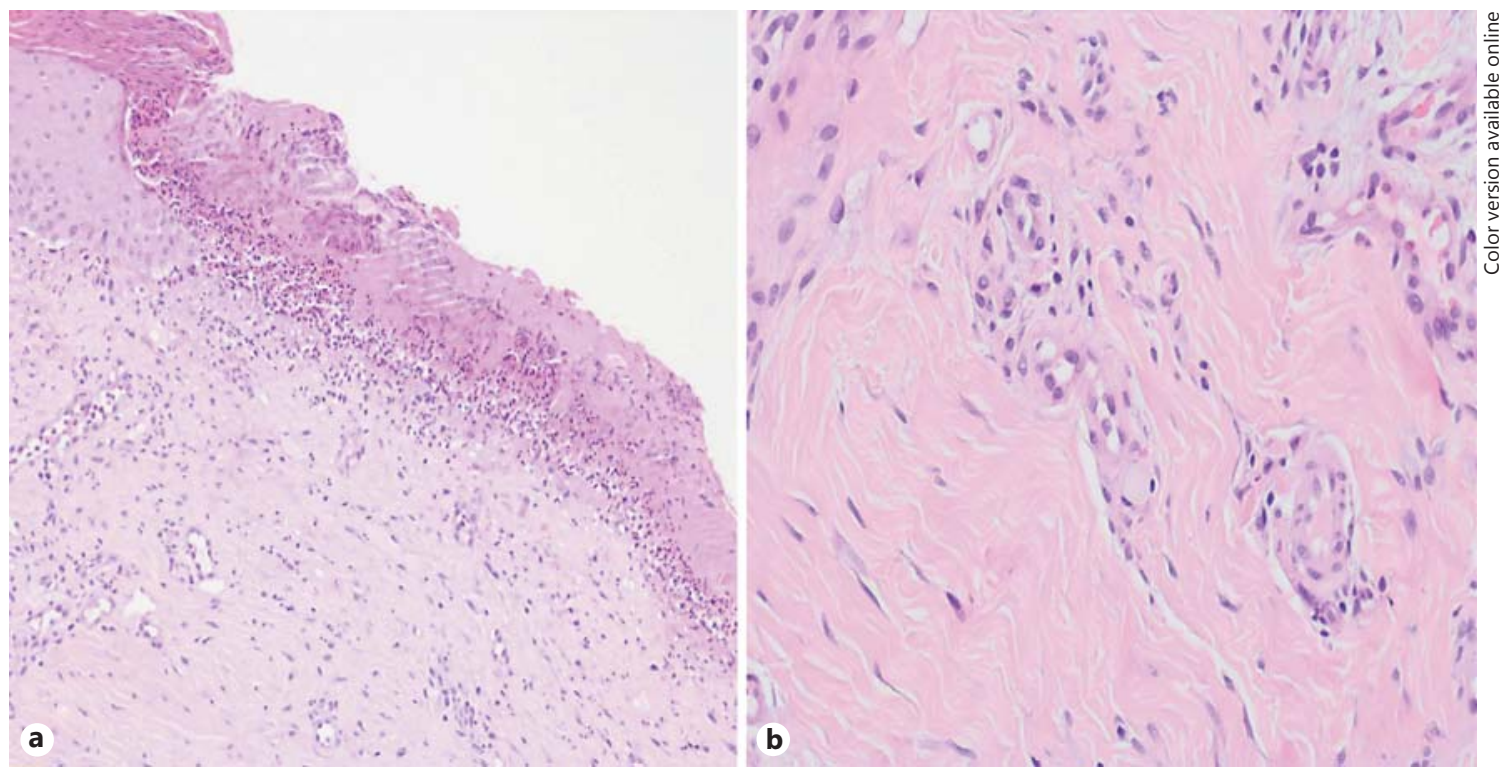

Fig. 2. Skin biopsies. a Area of ulceration covered by fibrin, locules of serum, and neutrophil debris. In the dermis, increased vessels presented together with lymphocytic inflammation and plasma cells. $\mathbf{b}$ Extensive fibrosis and only isolated follicles remained.

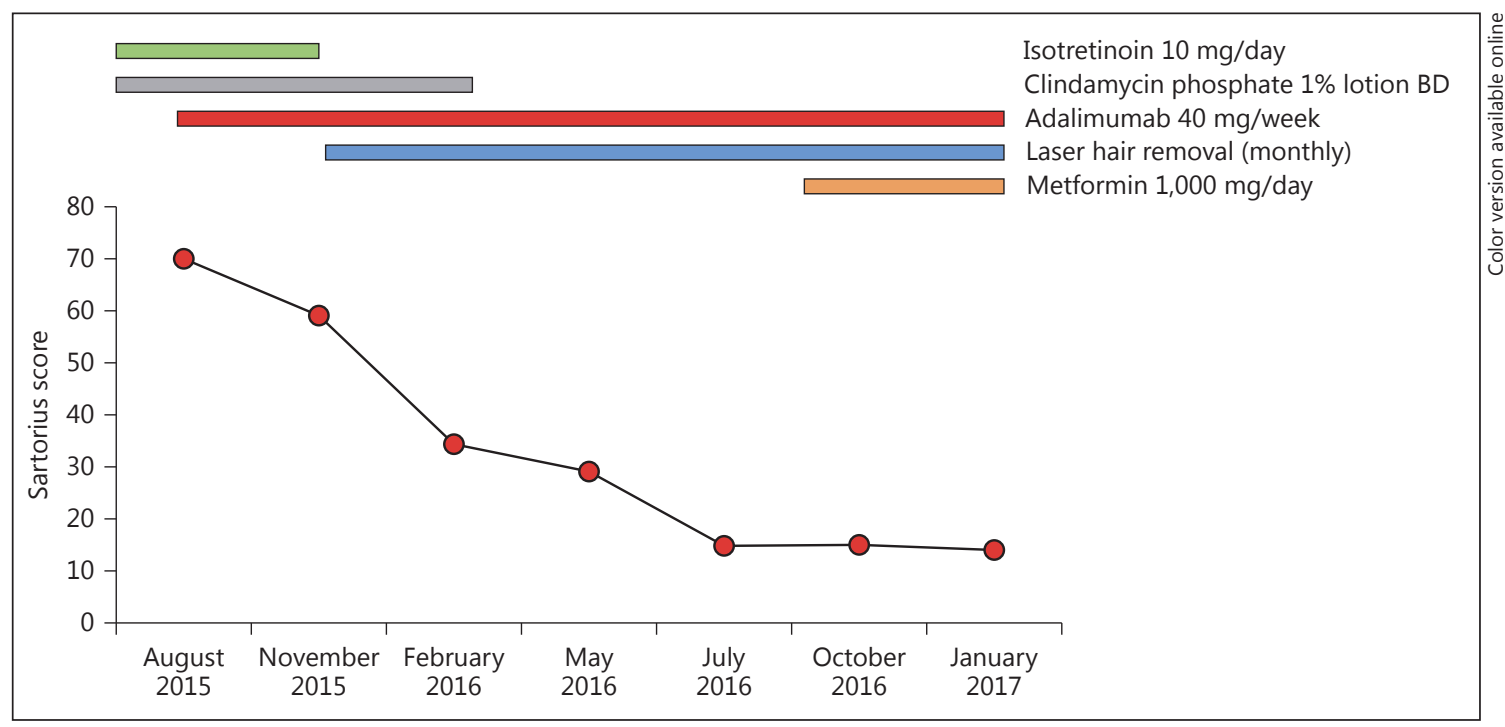

Fig. 3. Sartorius score evolution during adalimumab treatment. The graph illustrates the gradual decrease of Sartorius score during the treatment with adalimumab and laser hair removal.

\section{Discussion}

Atypical areas including the ears, face, posterior neck, chest, and back have been described in patients with HS in case reports and case series, especially in men [1-3]. These locations are infrequent, and most of the cases re- ported showed lesions of HS in unusual locations associated with other typical areas affected, which facilitates the diagnosis $[2,3]$. In this case, the diagnosis was not straightforward considering that the only parts of the body compromised were the anterior neck and jawline. The most important differential diagnoses considered were an in- 
fectious disease or acne conglobata. All the tests performed helped to rule out an infectious cause.

Some clinical features help to distinguish between HS and acne conglobata. Closed comedones are present in the latter and absent in the former, while the rope-like bridged scars are typical of HS and are different from the common hypertrophic or keloidal acne scars [3]. Thus, the clinical appearance of the lesions, the lack of response to isotretinoin, and a satisfactory response to adalimumab, one of the biologics with the strongest evidence to treat HS [4], allowed us to conclude that this patient has a localized form of HS involving an atypical location, instead of a severe type of acne. Moreover, the involvement of atypical locations such as the anterior neck and jawline (areas with an abundant number of terminal hair follicles) in this case supports another possible pathogenic theory of HS, which consists of the occlusion of terminal hair follicles rather than to be essentially a disorder of the apocrine glands [5-7]. The present case is relevant given that it helps clinicians to remember that HS may be isolated in atypical locations, such as the anterior aspect of the neck and chin. Early recognition of this atypical presentation of HS and treatment with a biological agent if the patient is not responding to standard therapy is important to prevent scarring.

\section{Acknowledgement}

Dr. M.A. Castrillón Velásquez was supported by an Australian government award, the Endeavour Scholarships and Fellowships, under the supervision of Professor Dedee F. Murrell.

\section{Statement of Ethics}

The patient has given informed consent for this case report to be published.

\section{Disclosure Statement}

None of the authors have conflicts of interest and financial disclosures.

\section{References}

1 Canoui-Poitrine F, Revuz JE, Wolkenstein P, et al: Clinical characteristics of a series of 302 French patients with hidradenitis suppurativa, with an analysis of factors associated with disease severity. J Am Acad Dermatol 2009; 61:51-57.

2 Syed ZU, Hamzavi IH: Atypical hidradenitis suppurativa involving the posterior neck and occiput. Arch Dermatol 2011;147:1343-1344.
3 Poli F, Wolkenstein P, Revuz J: Back and face involvement in hidradenitis suppurativa. Dermatology 2010;221:137-141.

4 Deckers IE, Prens EP: An update on medical treatment options for hidradenitis suppurativa. Drugs 2016;76:215-229.

5 Slade DE, Powell BW, Mortimer PS: Hidradenitis suppurativa: pathogenesis and management. Br J Plast Surg 2003;56:451-461.
6 Yu CCW, Cook MG: Hidradenitis suppurativa: a disease of follicular epithelium, rather than apocrine glands. Br J Dermatol 1990;122: 763-769.

7 Alikhan A, Lynch PJ, Eisen DB: Hidradenitis suppurativa: a comprehensive review. J Am Acad Dermatol 2009;60:539-561. 